\title{
Effect of vacancy defects on the electronic transport properties of an $\mathrm{Ag}-\mathrm{ZnO}-\mathrm{Pt}$ sandwich structure
}

\author{
G. R. Berdiyorov ${ }^{1}$ (I) F. Boltayev ${ }^{2} \cdot$ G. Eshonqulov ${ }^{3} \cdot$ H. Hamoudi ${ }^{1}$
}

Received: 26 July 2020 / Accepted: 23 January 2021 / Published online: 3 March 2021

(c) The Author(s) 2021

\begin{abstract}
The effect of zinc and oxygen vacancy defects on the electronic transport properties of $\mathrm{Ag}(100)-\mathrm{ZnO}(100)-\mathrm{Pt}(100)$ sandwich structures is studied using density functional theory in combination with the nonequilibrium Green's functional formalism. Defect-free systems show clear current rectification due to voltage dependent charge localization in the system as revealed in our transmission eigenstates analysis. Regardless of the location, oxygen vacancies result in enhanced current in the system, whereas Zn vacancy defects reduce the charge transport across the junction. The current rectification becomes less pronounced in the presence of both types of vacancy defects. Our findings can be of practical importance for developing metal-insulator-metal diodes.
\end{abstract}

Keywords Electronic transport · Green's function · Metal-ZnO-metal junctions

\section{Introduction}

Metal-insulator-metal (MIM) tunnel diodes, where two metal electrodes are separated by a nanoscale insulating layer (or multilayers) have a great potential for high-frequency applications such as rectenna-based energy harvesting [1-4], THz and IR detection [5, 6] and wireless power transmissions [7]. Ultrafast quantum tunneling of charge carriers through the insulating layer is the main process for the such high-frequency operations [8]. Ultralow capacitance is the main requirement for the high frequency applications MIM diodes, which can be achieved by, e.g.. increasing the thickness of the dielectric layer. However, the latter increases the diode resistance exponentially [9], which negatively affects the diode properties of the device.

Recent experiments show that MIM diodes with $\mathrm{ZnO}$ as an insulating layer provide smallest resistance with reasonable current rectification level [2]. The electrical conduction

$\triangle$ G. R. Berdiyorov

gberdiyorov@hbku.edu.qa

1 Qatar Environment and Energy Research Institute, Hamad Bin Khalifa University, Doha, Qatar

2 Tashkent University of Information Technologies, 100200 Tashkent, Uzbekistan

3 Department of Physics, National University of Uzbekistan, Tashkent, Uzbekistan properties of such junctions depend on the $\mathrm{ZnO}$ layer thickness and doping type/level [10]. Vacancy defects at the interfaces also play an important role in defining electronic and optical properties of metal-ZnO-metal junctions [11-13]. For example, creation/recovery of oxygen vacancies may result in optical and resistive switching in such MIM junctions [14]. Bipolar resistive switching in $\mathrm{Ag} / \mathrm{ZnO} / \mathrm{Pt}$ junction can also be related to charge carrier dynamics at $\mathrm{Zn}$ vacancy defects at the interfaces [15].

In this work, we use first-principles density functional theory (DFT) calculations in combination with the nonequilibrium Green's functional formalism to conduct quantum transport calculations in $\mathrm{Ag}-\mathrm{ZnO}-\mathrm{Pt}$ sandwich structures containing $\mathrm{O}$ and $\mathrm{Zn}$ vacancy defects (see Fig. $1 \mathrm{~b}, \mathrm{c}$ ). As a reference, we also study the electronic transport properties of defect free system (see Fig. 1a) which shows clear current rectifications due to the asymmetric coupling. The presence of $\mathrm{O}$ vacancy at the interfaces results in enhanced current rectification for both polarity of the applied voltage. On the contrary, $\mathrm{Zn}$ vacancies reduce the current through the junction which is in agreement with previous experimental findings [15]. For both types of vacancy defects, the rectification level is reduced in the system. The obtained results are explained in terms of geometry- and bias-dependent charge localization in the system as revealed in our transmission eigenstates analysis. We have also conducted density of states and transmission spectrum analysis to explain the 

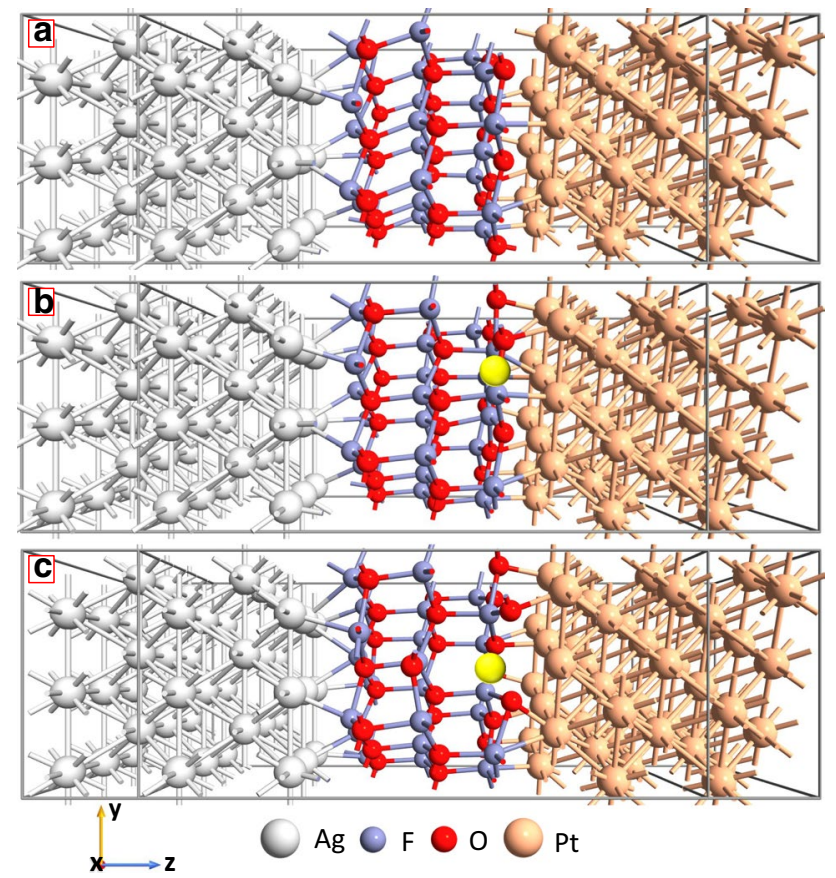

Fig. 1 Device geometries: $\mathrm{Ag}(100)-\mathrm{ZnO}(100)-\mathrm{Pt}(100)$ sandwich structure without defects (a) and with $\mathrm{O}$ vacancy (b) and $\mathrm{Zn}$ vacancy (c) defects. Yellow dots indicate the location of the vacancy defects. Periodic boundary conditions are applied along the $x$ - and $y$-directions, and the transport is calculated along the $z$-direction (Color figure online)

effect of vacancy defects on the electronic transport properties of the system.

\section{Model systems and computational details}

The optimized face-centered cubic structures of $\mathrm{Au}, \mathrm{Pt}$ and ZnO have lattice parameters $4.078 \AA$, 3.924 Aand $4.630 \AA$, respectively. Using these structures, we have created our reference system, which consists of $5.93 \AA$ thick $\mathrm{ZnO}$ (100) slab sandwiched between $6.1 \mathrm{~nm}$ thick Ag (100) and 5.92 $\mathrm{nm}$ Pt (100) slabs (see Fig. 1a). The interface area is 76.04 $\AA^{2}$, and the mean absolute strain is around $1 \%$. To study the effect of vacancy defects, we have created either single atom $\mathrm{Zn}$ (Fig. $1 \mathrm{~b}$ and panel 2 in Fig. 6a) or O (Fig. 1c and panel 1 in Fig. 6a) vacancies at the $\mathrm{ZnO}-\mathrm{Pt}$ (Fig. 1) and $\mathrm{Ag}-\mathrm{ZnO}$ (Fig. 6) interfaces. All three systems are geometry optimized using DFT within the generalized gradient approximation of Perdew-Burke-Ernzerhof (PBE) [16] for the exchangecorrelation. Brillouin zone integration is performed using Monkhorst-Pack scheme [17] and non-bound van der Waals interactions are taken into account using Grimme's PBE empirical dispersion correction [18]. Double-zetapolarized basis sets of local numerical orbitals are used as implemented in the norm-conserving Fritz-Haber Institute
(FHI) pseudopotential. LBFGS method is applied for the structural optimizations with the convergence criterion for Hellman-Feynman forces as $0.01 \mathrm{eV} / \AA$. The geometry optimization resulted in strong covalent bonding between the atoms at both $\mathrm{Ag}-\mathrm{ZnO}$ and $\mathrm{ZnO}-\mathrm{Pt}$ interfaces (see Fig. 1 and panels 1 and 2 in Fig. 6). Two probe device geometries are constructed with the size of the electrodes $4.09 \AA$ and $3.92 \AA$, respectively, for $\mathrm{Ag}$ and Pt electrodes.

The electronic transport calculations are performed using the nonequilibrium Green's function formalism [19] using $6 \times 6 \times 251 k$-points. The current-voltage $(I-V)$ characteristics are calculated using the Landauer-Büttiker expresion [20]:

$I(V)=\frac{2 e}{h} \int_{\mu_{L}}^{\mu_{R}} T(E, V)\left[f\left(E-\mu_{L}\right)-f\left(E-\mu_{R}\right)\right] \mathrm{d} E$,

where $T(E, V)$ is the transmission spectrum for the given bias voltage $(V), f\left(E, E_{F}\right)$ is the Fermi-Dirac distribution function, and $\mu_{L} / \mu_{R}$ is the chemical potential of the left/right electrode. All the calculations are conducted using the computational package Atomistix toolkit [21-23].

\section{Results and discussion}

We start with calculating the formation energy of single $\mathrm{Zn}$ and $\mathrm{O}$ vacancy defects at both the $\mathrm{ZnO}-\mathrm{Pt}$ (Fig. 1) and $\mathrm{Ag}-\mathrm{ZnO}$ (Fig. 6) interfaces using the following formula:

$E_{v}^{i}=E^{\text {interface }}-\left(E^{\text {interface-atom }}-E^{i}\right)$,

where $E^{\text {interface }}$ is the total energy of the interface, $E^{\text {interface-vacancy }}$ is the total energy of the interface with single atom vacancy, and $E^{i}$ is the calculated cohesive energy. For $\mathrm{Zn}$ atom, we have taken the hexagonal bulk $\mathrm{Zn}$ structure and for $\mathrm{O}$ we used $\mathrm{O}_{2}$ molecule. Table 1 shows the calculated values of the formation energies. The results show that the formation energies depend on the type of the vacancy and on the interfaces; the smallest formation energy is obtained for $\mathrm{O}$ vacancy at $\mathrm{Ag}-\mathrm{ZnO}$ interface, whereas the largest energy is obtained for $\mathrm{Zn}$ vacancy at the same interface.

As a main result, we show in Fig. 2a the current-voltage characteristics of the considered systems for the voltage range from -2 to $2 \mathrm{~V}$. For the considered thickness of the $\mathrm{ZnO}$ layer (5.93 $\mathrm{A}$ ), finite current is obtained for any value of the applied bias for all three systems due to electron tunneling mechanism. The current in all systems increases monotonically with further increasing the applied voltage. The presence of the $\mathrm{O}$ vacancy defect increases the current through the junction (compare solid-black and open-red circles). The current enhancement is more pronounced at small biases (more than 50\%) and with further increasing the bias voltage the current values in both systems become equal. On the contrary, the current in the system with $\mathrm{Zn}$ vacancy 

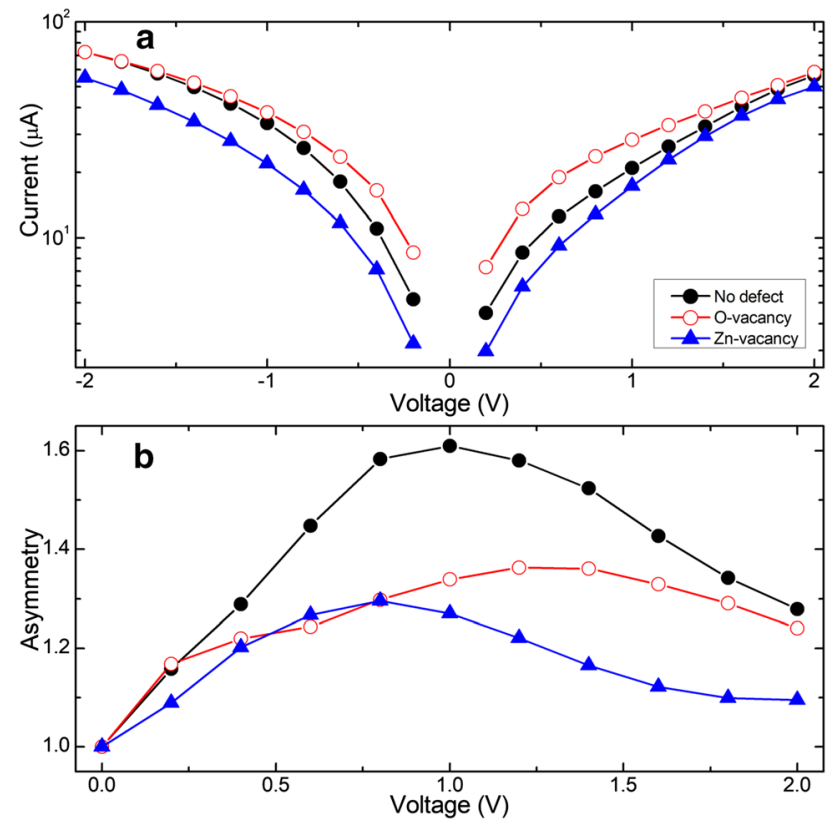

Fig. 2 Current voltage characteristics (a) and asymmetry (b) of the $\mathrm{Ag}-\mathrm{ZnO}-\mathrm{Pt}$ sandwich structure without defects (solid-black circles) and with $\mathrm{O}$ (open-red circles) and $\mathrm{Zn}$ (solid-blue triangles) vacancy defects (Color figure online)

Table 1 Calculated formation energies of single $\mathrm{Zn}$ and $\mathrm{O}$ vacancy defects at $\mathrm{ZnO}-\mathrm{Pt}$ and $\mathrm{Ag}-\mathrm{ZnO}$ interfaces

\begin{tabular}{lll}
\hline Interface & Zn vacancy $(\mathrm{eV})$ & O vacancy $(\mathrm{eV})$ \\
\hline $\mathrm{ZnO}-\mathrm{Pt}$ & -3.54 & -3.06 \\
$\mathrm{Ag}-\mathrm{ZnO}$ & -2.39 & -5.04 \\
\hline
\end{tabular}

defect (filled-blue triangles) is smaller as compared to the current in the pristine sample for all considered bias voltages. The effect of vacancy defects on the resistive switching properties of $\mathrm{Ag}-\mathrm{ZnO}-\mathrm{Pt}$ junctions has been studied experimentally in Ref. [15]. Reduced current has been obtained after post annealing in oxygen ambience. As verified by deep level defect emission, the post-annealing in oxygenrich environment favors the creation of $\mathrm{Zn}$ vacancies and annihilation of $\mathrm{O}$ vacancies. Therefore, the reduced current after the annealing can be related to the increased number of $\mathrm{Zn}$ vacancies and reduced $\mathrm{O}$ vacancies. In this respect, our findings are in good agreement with the experimental results of Ref. [15].

All systems present asymmetric $I-V$ characteristics with reverse current (i.e. current at negative voltages) being larger than the forward one. Therefore, we defined the asymmetry (i.e. current rectification level) as the ratio of the reverse current to the forward current. Figure $2 b$ shows the asymmetry of the $I-V$ curves as a function of the bias voltage. For the defect-free system, the asymmetry increases with increasing the applied voltage and reaches maximum at $1 \mathrm{~V}$ (see soolidblack circles in Fig. 2b). For the considered thickness of the $\mathrm{ZnO}$ layer maximum rectification level of $60 \%$ is obtained. Recent simulation results show that the asymmetry of metalinsulator-metal junctions increase linearly with increasing the thickness of the insulating layer [24]. The asymmetry decreases further with increasing the applied voltage. The presence of both types of defects reduces the current rectification in the system (see open-red circles and filled-blue triangles in Fig. 2b). The maximum rectification levels are $36 \%$ and $30 \%$, respectively, for $\mathrm{O}$ and $\mathrm{Zn}$ vacancies. The location of the maximum asymmetry also changes with introducing the vacancy defects: it shift for larger voltages for O-vacancy, whereas Zn-vacancy shifts the maximum towards smaller bias voltages.

To find the origin for the changes in the electronic transport properties of the considered samples due to the vacancy defects, we have calculated and analyzed the transmission spectra and device density of states (DDOS) of the considered systems. Figure 3 shows zero-bias DDOS (a) and transmission spectra (b) of the system without defect (solidblack curves), with O-vacancy (dashed-red curves) and with Zn-vacancy (dotted-blue curves) defects. For the considered range of electron energy, all three systems show very similar DDOS, except some resonance peaks. However, the presence of the vacancy defects changes transmission spectrum of the system considerably. For example, the O-vacancy defect increases the electron transmission, whereas the Zn-vacancy results in reduced transmission as compared to the transmission of the reference system. These results explain the obtained changes in the $I-V$ characteristics, i.e., enhanced current for $\mathrm{O}$ vacancy defects and current reduction for $\mathrm{Zn}$ vacancy defect (see Fig. 2a). In order to get further insight into the conductivity changes due to the vacancy defects, we have calculated the molecular projected self-consistent Hamiltonian (MPSH) eigenstates for different electron energies. Panels 1-6 in Fig. 3 show the isosurface plots of the MPSH eigenstates for the states below (panels 1, 3 and 5) and above (panels 2, 4 and 6) the Fermi level. Those states are highlighted by symbols $1-6$ on the transmission curves in Fig. 3b. For all three systems, the MPSH states below the Fermi level are extended through the junction which will increase the transmission probabilities of the charge carriers. For the state above the Fermi level, delocalization of the electronic states is obtained only for the system with O-vacancy defect (panel 4), which explains the enhanced transmission for this system (dashed-red curve in Fig. 3b). For the other two systems, the considered states above the Fermi level are localized on the Pt part of the junction (panels 2 and 6). The localization is more pronounced in the system with Zn-vacancy (panel 6). Therefore, we obtained the lowest transmission for the latter sample (dotted-blue curve in Fig. 3b). Thus, the nanoscale charge localization can be 

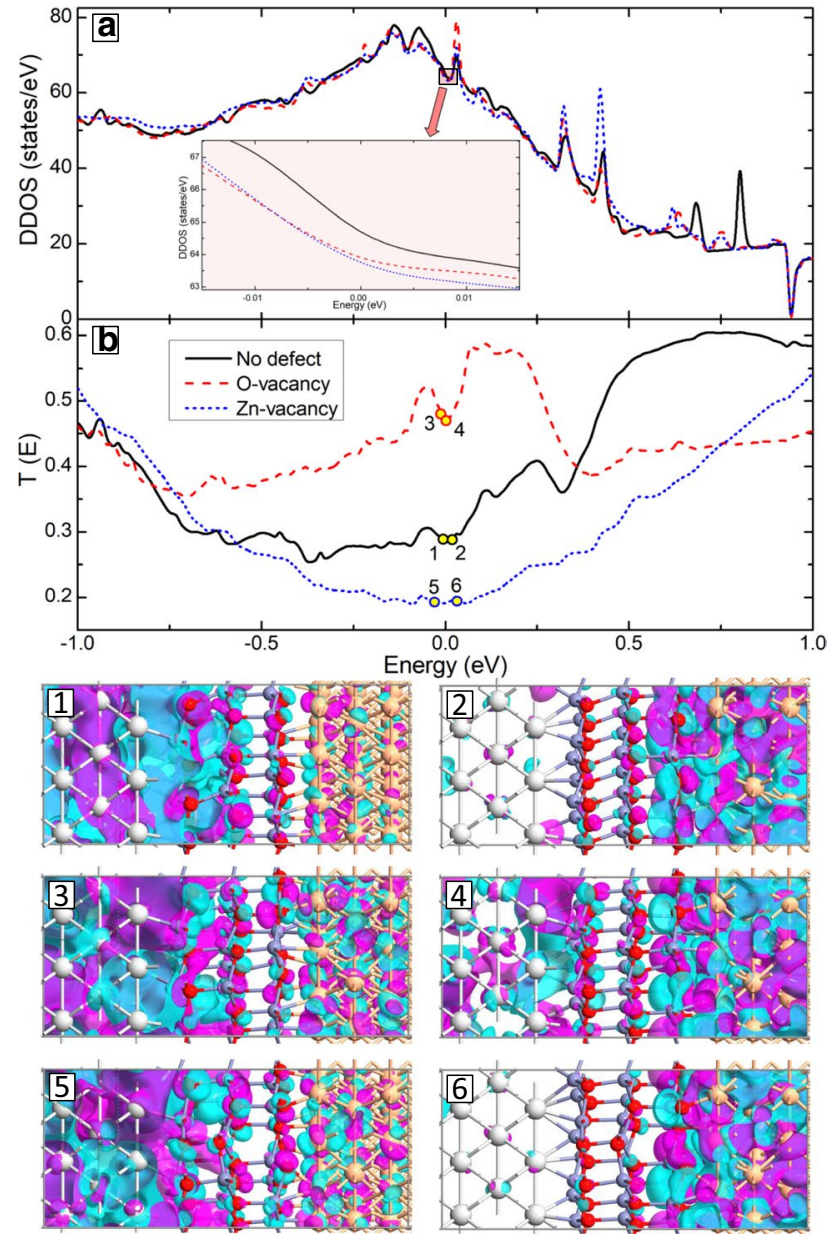

Fig. 3 Zero bias device density of states (a) and transmission spectra (b) as a function of electron energy (zero corresponds to Fermi energy) for the $\mathrm{Ag}-\mathrm{ZnO}-\mathrm{Pt}$ sandwich structure without (solid-black curves), with $\mathrm{O}$ vacancy (dashed-red curves) and with $\mathrm{Zn}$ vacancy (dotted-blue curves). Inset in a shows the enlargement of DDOS near the Fermi level. Panels $1-6$ show the isosurface plots (isovalues \pm $0.015 \AA^{-3 / 2}$ ) of the molecular projected self-consistent Hamiltonian states obtained at the electron energy values indicated on the transmission curves (Color figure online)

used to explain the effect of vacancy defects on the transport properties of the $\mathrm{Ag}-\mathrm{ZnO}-\mathrm{Pt}$ junctions. Another factor that affects the current through the system is the electrostatic potential profile along the transport direction. To see the effect of the vacancy defects on the electrostatic potential variations, we have conducted electrostatic potential calculations for the systems in Fig. 1. The obtained results are shown in Fig. 4. In all cases, we obtained identical potential oscillations inside the electrode areas. Clear oscillations are also obtained inside the $\mathrm{ZnO}$ region. However, the amplitude of the oscillations in this region depends on the type of the vacancy defects. For example, in the case of the $\mathrm{Zn}$ vacancy defects the amplitude of the potential variations becomes larger as compared to the reference system (compare

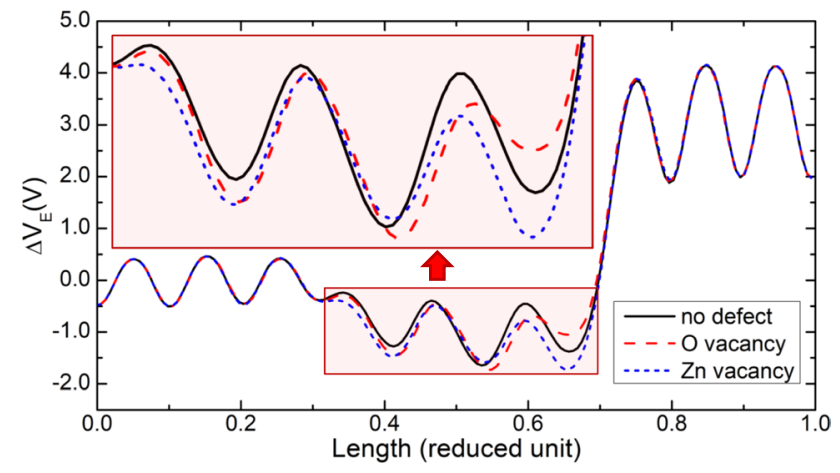

Fig. 4 Variations of the electrostatic difference potential along the transport direction at zero voltage biasing for the $\mathrm{Ag}-\mathrm{ZnO}-\mathrm{Pt}$ sandwich structure without defects (solid-black curve) and with $\mathrm{O}$ (dashed-red curve) and $\mathrm{Zn}$ (dotted-blue curve) vacancy defects (Color figure online)

solid-black and dotted-blue curves in Fig. 4). Such electrostatic potential peaks increase the back-scattering of the electrons and decrease their transmission probability through the system. This explains the reduced current in the system in the presence of the $\mathrm{Zn}$ vacancy defect (see Fig. 2a). On the contrary, the introduction of the oxygen vacancy defect reduces the amplitude of the electrostatic potential variations (see dashed-red curve in Fig. 4), which in turn increases the transmission probability of the electrons. Thus, electrostatic potential profile can also be used to explain the transport properties of $\mathrm{Ag}-\mathrm{ZnO}-\mathrm{Pt}$ sandwich structures due to the presence of the vacancy defects.

We also used the transmission and MPSH analyses to explain the current rectification in the considered samples. As an example, we present in Fig. 5 transmission spectra of the defect-free system for negative (solid-back curve) and positive (dashed-red curve) bias voltages together with isosurface plots of the MPSH states at electron energies indicated on the transmission curves. For the negative voltage bias, both of the states below (panel 1) and above (panel 2) the Fermi level are extended over the junction, and therefore, electrons have larger transmission in that range of electron energies (solid-black curve in Fig. 5a). On the contrary, for the positive voltage biasing, the states above the Fermi level (panels 3 and 4) are localized at the right electrode part of the junction. Consequently, the probability of the charge carriers is strongly reduced (see dashed-red curve in Fig. 5a). Thus, the current rectification in the considered systems can be explained by voltagepolarity dependent charge localization in the system.

In the above systems, the vacancy defects are created at the $\mathrm{ZnO}(100)-\mathrm{Pt}(100)$ interface (see Fig. 1). Figure 6 shows the results when the vacancy defects are created at the $\mathrm{Ag}(100)-\mathrm{ZnO}(100)$ interface (see the insets in Fig. 6a 

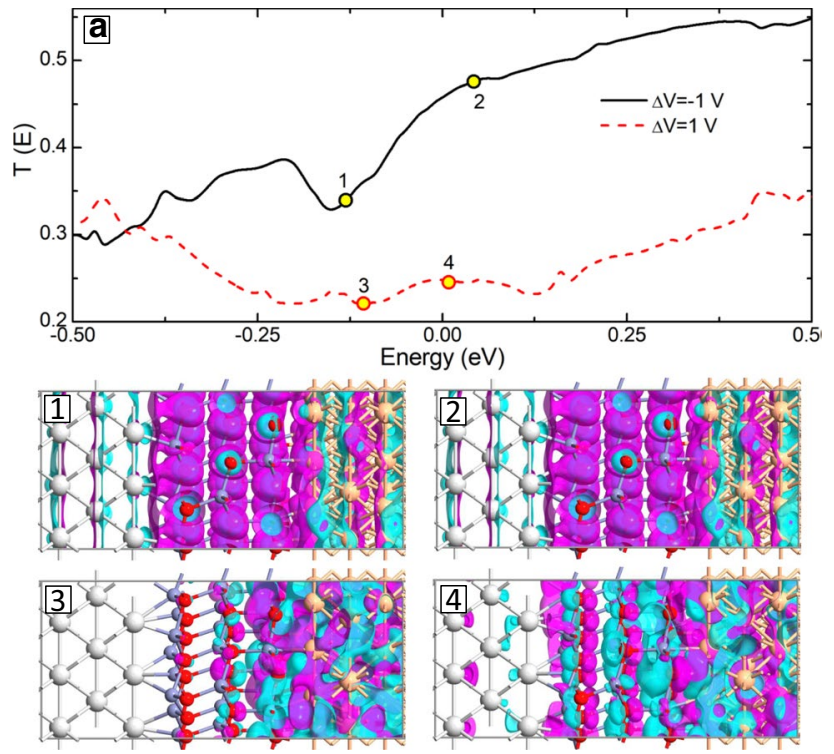

Fig. 5 a Transmission spectra of the defects-free sample for bias voltage $\Delta V=-1 \mathrm{~V}$ (solid-black curve) and $\Delta V=1 \mathrm{~V}$ (dashed-red curve). Panels $1-8$ show the isosurface plots (isovalues $\pm 0.015 \AA^{-3 / 2}$ ) of the eigenstates corresponding to the electron energies indicated on the transmission curves. The results are present both for negative (panels $1-4$ ) and positive (panels 5-8) bias voltages (Color figure online)
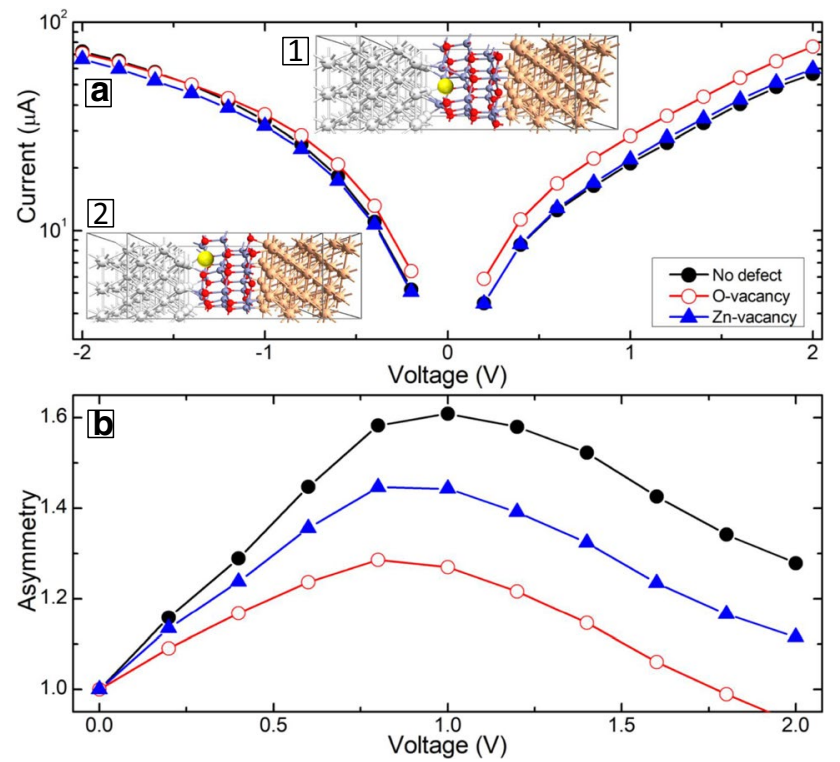

Fig. 6 Current voltage characteristics (a) and asymmetry (b) of the $\mathrm{Ag}-\mathrm{ZnO}-\mathrm{Pt}$ sandwich structure without defects (solid-black circles) and with $\mathrm{O}$ (open-red circles) and $\mathrm{Zn}$ (solid-blue triangles) vacancy defects. Insets show the device geometries with $O$ vacancy (1) and $\mathrm{Zn}$ vacancy (2) defects. The defects are created near the Ag electrode (Color figure online)

for the device geometries). Qualitatively similar results are obtained also for these vacancy defects:
- The O-vacancy defect results in enhanced current for both polarity of the applied bias (open-red circles in Fig. 6a).

- The current in the system with the Zn-vacancy defect becomes smaller for negative biases and slightly larger for the positive voltages (filled-blue triangles).

- The presence of both types of defects results in reduced current rectification (Fig. 6b) with rectification level for the $\mathrm{Zn}$ vacancy being larger than the one for the $\mathrm{O}$ vacancy.

Figure 7 shows the zero-bias DDOS (a) and transmission spectra (b) of the system without defect (solid-black curves), with O-vacancy (dashed-red curves) and with $\mathrm{Zn}$ vacancy (dotted-blue curves) defects, where the defects are created at the $\mathrm{Ag}(100)-\mathrm{ZnO}(100)$ interface. It is seen from this figure that the reference system and the sample with $\mathrm{Zn}$ vacancy show similar DDOS and transmissions spectra (see solid-black and dotted-blue curves). Therefore, we obtained the similar current in these two systems (see Fig. 6a). The system with $\mathrm{O}$ vacancy shows slightly higher DDOS at the Fermi level (see the inset of Fig. 7a) and significantly larger transmission for the considered range of the electron energy (dashed-red curves in Fig. 7b). The latter results in larger current through the system.

As seen from the calculated $I-V$ curves (see Fig. $6 \mathrm{a}$ ), the effect of the vacancy defects becomes more pronounced in terms of current value for positive voltage biases. To understand these changes, we have calculated and analyzed

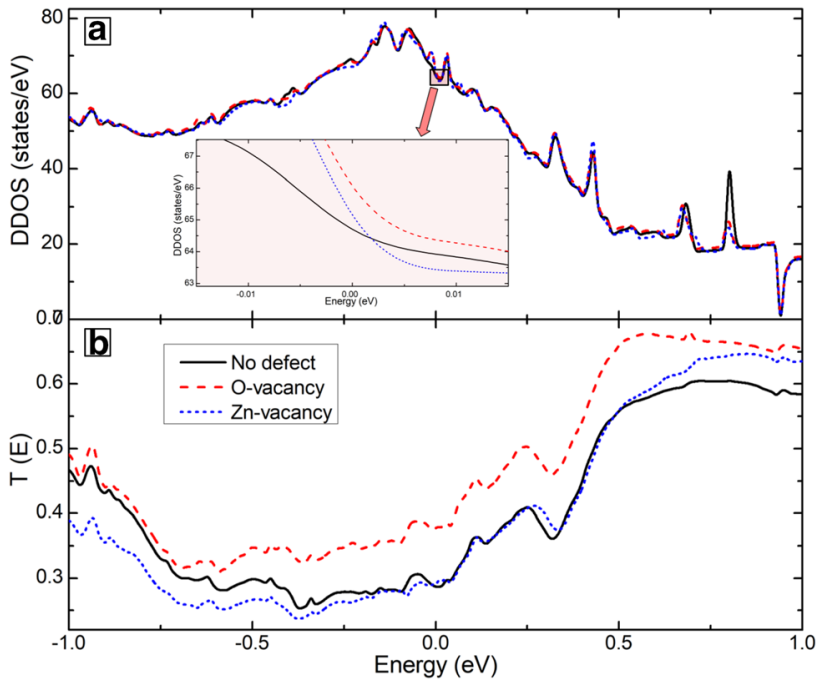

Fig. 7 Zero bias device density of states (a) and transmission spectra (b) as a function of electron energy for the $\mathrm{Ag}-\mathrm{ZnO}-\mathrm{Pt}$ sandwich structure in Fig. 6 without (solid-black curves), with $\mathrm{O}$ vacancy (dashed-red curves) and with $\mathrm{Zn}$ vacancy (dotted-blue curves). Inset in a shows the enlargement of DDOS near the Fermi level (Color figure online) 


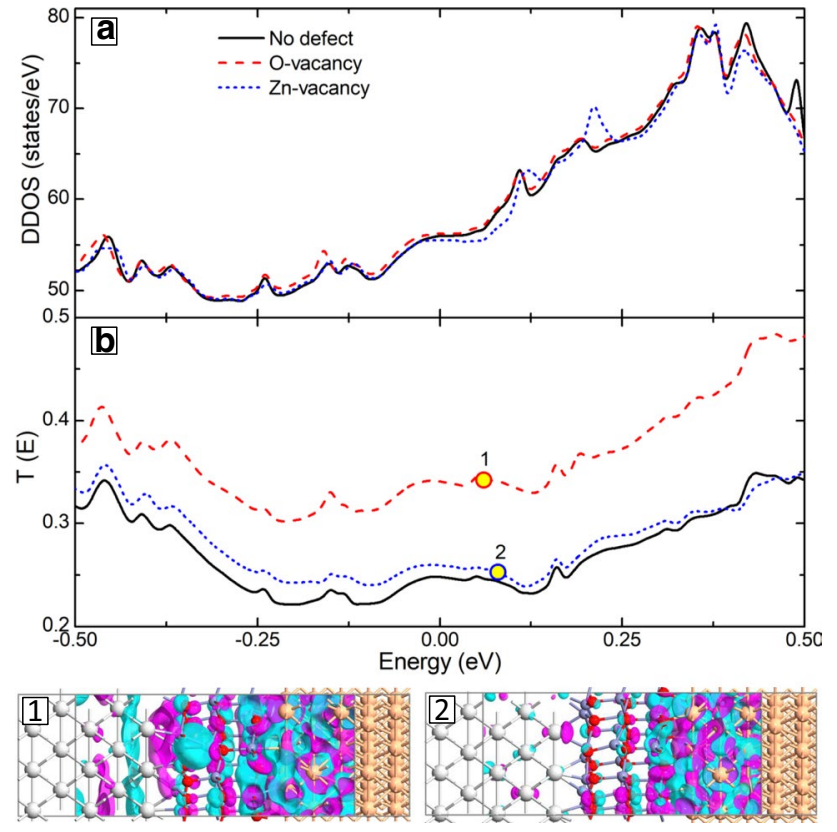

Fig. 8 Device density of states (a) and transmission spectra (b) at bias voltage $\Delta V=1 \mathrm{~V}$ as a function of electron energy for the $\mathrm{Ag}-$ $\mathrm{ZnO}-\mathrm{Pt}$ sandwich structure in Fig. 6 without (solid-black curves), with $\mathrm{O}$ vacancy (dashed-red curves) and with $\mathrm{Zn}$ vacancy (dottedblue curves). Panels $1-2$ show the isosurface plots (isovalues \pm 0.015 $\AA^{-3 / 2}$ ) of the eigenstates corresponding to the electron energies indicated on the transmission curves (Color figure online)

DDOS and transmission spectra of the considered systems at finite positive voltage biasing. Figure 8 shows the results for voltage $\Delta V=1 \mathrm{~V}$. As in the case of zero voltage biasing (see Fig. 7a), all three systems show similar DDOS for the considered range of the electron energy, which is used for the integration in Eq. 1. However, the transmission spectra of the defective systems become larger as compared to the reference system (see Fig. 8b). This enhancement is due to the delocalization of the electronic states as revealed in our MPSH eigenstates analysis (see panels 1 and 2 in Fig. 8).

\section{Conclusions}

Quantum transport calculations are conducted to study the effect of vacancy defects on the electronic transport properties of $\mathrm{Ag}-\mathrm{ZnO}-\mathrm{Pt}$ sandwich structures. Defect-free systems show clear current rectification with maximum rectification level of 1.6 for the considered sample parameters. The presence of $\mathrm{O}$ vacancy defects results in current enhancement in the system, whereas the $\mathrm{Zn}$ vacancies reduce the electronic transport through the junction, except for larger positive voltage biasing when the defect is created at $\mathrm{Ag}-\mathrm{ZnO}$ interfaces. Regardless of the type and location of the defects, the current rectification level decreases as compared to the defect-free sample. The obtained results are explained by nanoscale-charge localization in the system as revealed in our MPSH eigenstates analysis. These findings can be useful for developing $\mathrm{ZnO}$-based metal-insulator-metal diodes.

Acknowledgements Authors also acknowledge the usage of computer facilities of Qatar Environment and Energy Research Institute.

Funding Open access funding provided by the Qatar National Library.

Open Access This article is licensed under a Creative Commons Attribution 4.0 International License, which permits use, sharing, adaptation, distribution and reproduction in any medium or format, as long as you give appropriate credit to the original author(s) and the source, provide a link to the Creative Commons licence, and indicate if changes were made. The images or other third party material in this article are included in the article's Creative Commons licence, unless indicated otherwise in a credit line to the material. If material is not included in the article's Creative Commons licence and your intended use is not permitted by statutory regulation or exceeds the permitted use, you will need to obtain permission directly from the copyright holder. To view a copy of this licence, visit http://creativecommons.org/licenses/by/4.0/.

\section{References}

1. Sharma, A., Singh, V., Bougher, T.L., Cola, B.A.: A carbon nanotube optical rectenna. Nat. Nanotechnol. 10, 1027-1032 (2015)

2. Anderson, E.C., Bougher, T.L., Cola, B.A.: High performance multiwall carbon nanotube-insulator-metal tunnel diode arrays for optical rectification. Adv. Electron. Mater. 4, 1700446 (2018)

3. Matsuura, D., Shimizu, M., Yugami, H.: High-current density and high-asymmetry MIIM diode based on oxygen-non-stoichiometry controlled homointerface structure for optical rectenna. Sci. Rep. 9, 19639 (2019)

4. Berdiyorov, G.R., Hamoudi, H.: Doping-enhanced current rectification in carbon nanotube-metal junctions for rectenna applications. ACS Omega 5, 189-196 (2020)

5. Hobbs, P.C.D., Laibowitz, R.B., Libsch, F.R.: NiNiONi tunnel junctions for terahertz and infrared detection. Appl. Opt. 44, 6813-6822 (2005)

6. Grover, S., Dmitriyeva, O., Estes, M.J., Moddel, G.: Travellingwave metal/insulatormetal diodes for improved infrared bandwidth and efficiency of antenna-coupled rectifiers. IEEE Trans. Nanotechnol. 9, 716-722 (2010)

7. Donchev, E., Pang, J.S., Gammon, P.M., Centeno, A., Xie, F., Petrov, P.K., Breeze, J.D., Ryan, M.P., Riley, D.J., Alford, N.M.: The rectenna device: from theory to practice (a review). MRS Energy Sustain. 1, E1 (2014)

8. Hartman, T.E.: Tunneling of a wave packet. J. Appl. Phys. 33, 3427 (1962)

9. Cowell, E.W., Alimardani, N., Knutson, C.C., Conley, J.F., Keszler, D.A., Gibbons, B.J., Wager, J.F.: Advancing MIM electronics: amorphous metal electrodes. Adv. Mater. 23, 74 (2011)

10. Li, L., Rees, P.: Quantum resonance of nanometre-scale metal$\mathrm{ZnO}-\mathrm{metal}$ structure and its application in sensors. AIP Adv. 6, 015003 (2016)

11. Vlasenko, L.S., Watkins, G.D.: Optical detection of electron paramagnetic resonance for intrinsic defects produced in $\mathrm{ZnO}$ by 2.5$\mathrm{MeV}$ electron irradiation in situ at 4.2 K. Phys. Rev. B 72, 035203 (2005) 
12. Brillson, L.J., Mosbacker, H.L., Hetzer, M.J., Strzhemechny, U., Jessen, G.H., Look, D.C., Cantwell, G., Zhang, J., Song, J.J.: Dominant effect of near-interface native point defects on $\mathrm{ZnO}$ Schottky barriers. Appl. Phys. Lett. 90, 102116 (2007)

13. Dong, Y., Tuomisto, F., Svensson, B.G., Kuznetsov, A.Y., Brillson, L.J.: Vacancy defect and defect cluster energetics in ionimplanted ZnO. Phys. Rev. B 81, 081201 (2010)

14. Yalishev, V.S., Kim, Y.S., Park, B.H.: Yuldashev, ShU: Resistance states dependence of photoluminescence in $\mathrm{Ag} / \mathrm{ZnO} / \mathrm{Pt}$ structures. Appl. Phys. Lett. 99, 012101 (2011)

15. Yalishev, V.S., Yuldashev, S.U., Kim, Y.S., Park, B.H.: The role of zinc vacancies in bipolar resistance switching of $\mathrm{Ag} / \mathrm{ZnO} / \mathrm{Pt}$ memory structures. Nanotechnology 23, 375201 (2012)

16. Perdew, J.P., Burke, K., Ernzerhof, M.: Generalized gradient approximation made simple. Phys. Rev. Lett. 77, 3865 (1996)

17. Monkhorst, H.J., Pack, J.D.: Special points for Brillouin-zone integrations. Phys. Rev. B 13, 5188 (1976)

18. Grimme, S.: Semiempirical GGA type density functional constructed with a longrange dispersion correction. J. Comp. Chem. 27, 1787 (2006)

19. Brandbyge, M., Mozos, J.L., Ordejón, P., Taylor, J., Stokbro, K.: Density-functional method for nonequilibrium electron transport. Phys. Rev. B 65, 165401 (2002)

20. Büttiker, M., Imry, Y., Landauer, R., Pinhas, S.: Generalized many-channel conductance formula with application to small rings. Phys. Rev. B 31, 6207 (1985)
21. QuantumATK 2019, Synopsys QuantumATK. https://www.synopsys.com/silicon/quantumatk.html

22. Smidstrup, S., Markussen, T., Vancraeyveld, P., Wellendorff, J., Schneider, J., Gunst, T., Verstichel, B., Stradi, D., Khomyakov, P.A., Vej-Hansen, U.G.: QuantumATK: an integrated platform of electronic and atomic-scale modelling tools. J. Phys. Condens. Matter 32, 015901 (2020)

23. Smidstrup, S., Stradi, D., Wellendorff, J., Khomyakov, P.A., VejHansen, U.G., Lee, M.-E., Ghosh, T., Jonsson, E., Jonsson, H., Stokbro, K.: First-principles Green's-function method for surface calculations: a pseudopotential localized basis set approach. Phys. Rev. B 96, 195309 (2017)

24. Berdiyorov, G.R., Hamoudi, H.: Effect of insulator thickness on the electronic transport through $\mathrm{CNT}-\mathrm{HfO}_{2}-\mathrm{Au}$ junction. Surf. Interfaces 22, 100823 (2020)

Publisher's Note Springer Nature remains neutral with regard to jurisdictional claims in published maps and institutional affiliations. 